\section{Incidence and outcome of no flow after primary percutaneous coronary intervention in acute myocardial infarction}

\author{
Goutam Datta* \\ Department of Cardiology, Burdwan Medical College and Hospital, West Bengal, India
}

\section{Abstract}

Background: Primary percutaneous coronary intervention $(\mathrm{PCl})$ of the infarct-related artery (IRA) is the most effective treatment modality in ST-segment elevation myocardial infarction (STEMI). Incidence of no flow is $8.8-10 \%$ in primary PCI of STEMI patients. Our aim was to study actual incidence and outcome of no flow patients.

Methods: Five hundred and eighty primary $\mathrm{PCl}$ patients were enrolled and evaluated from 2016 January to 2017 December.

We used drug eluting stents in all cases. Majority of our patients (>90\%) presented to emergency six hours after onset of symptoms. There were many patients where there was no flow even after mechanical thrombus aspiration and pharmacological vasodilator therapy. We have studied primary outcome (mortality) of no flow in those patients.

Results: There were 44 cases of no flow in our series (7.75\%). Involvement of Left anterior descending artery (LAD) was in eighteen patients. Right coronary artery (RCA) was culprit in twenty four cases. Only two cases were seen in LCX territory. One month mortality rate in no flow group was $50 \%$ and $6.25 \%$ in successful recanalization group. One year mortality was $12.5 \%$ in successful recanalization group and $66 \%$ in no flow group.

Conclusion: Refractory no flow during STEMI intervention is associated with increased incidence of major adverse cardiovascular events (MACE).

There is no established strategy to solve this phenomenon.

\section{More Information}

*Address for Correspondence: Goutam Datta, MD. DNB. DM. MRCP (UK), Associate Professor, Department of Cardiology, Burdwan Medical College and Hospital, West Bengal, P3 Lake Gardens Govt. Housing, 48/4 Sultan Alam Road, Calcutta 33, India, Tel: 9433415086 ; Email: goutamdattadn@yahoo.in

Submitted: 25 July 2020

Approved: 28 August 2020

Published: 31 August 2020

How to cite this article: Datta G. Incidence and outcome of no flow after primary percutaneous coronary intervention in acute myocardial infarction. J Cardiol Cardiovasc Med. 2020; 5: 153-156.

DOI: 10.29328/journal.jccm.1001102

Copyright: $\odot 2020$ Datta G. This is an open access article distributed under the Creative Commons Attribution License, which permits unrestricted use, distribution, and reproduction in any medium, provided the original work is properly cited.

Keywords: Primary PCl; No flow; Mortality

Check for updates

open Access

\section{Introduction}

Acute myocardial infarction is due to plaque rupture and thrombotic occlusion of epicardial coronary artery. Primary percutaneous coronary intervention of infarct related artery (IRA) is the most effective treatment modality in ST-segment elevation myocardial infarction (STEMI). Early revascularization of IRA will lead to favorable myocardial remodeling. It will reduce size of the infarct, improve left ventricular systolic and diastolic function and thereby will reduce MACE rate. Incidence of no flow is $\sim 8.8-10 \%$ in primary PCI of STEMI patients and it is associated with different risk factors like advanced age, delayed presentation, ectatic coronary artery and huge thrombus burden [1].

No flow after primary PCI may occur due to incomplete stent expansion, vaso spasm, dissection or in situ thrombosis. Microvascular obstruction and distal embolization are other contributing factors. Clinically no flow may present with the recurrence of chest pain, heart failure, malignant ventricular arrhythmias and cardiogenic shock. Angiographic no flow after PCI is associated with reduced myocardial salvage, larger infarct size and adverse cardiac remodeling. Early detection, preventive measures and treatment of no flow will decide final outcome after primary PCI.

\section{Methods}

Patients in the age group of 18 to 80 with STEMI were only considered for our study. No flow was defined by Thrombolysis in myocardial infarction (TIMI) grade zero or TIMI 1 flow. Our centre is a tertiary care university hospital which caters almost five districts of our state. 
Five hundred and eighty primary PCI patients were enrolled and evaluated from 2016 January to 2017 December. Ethical approval was taken and the study protocol was consistent with the ethical guidelines of the 1975 Declaration of Helsinki. Informed consents from all patients were part of study protocol. Majority of our patients ( $>90 \%$ ) came 6 hours after onset of chest pain. Average presentation was 8-12 hrs after onset of chest pain. Protocol guided periprocedural medication include Aspirin $(325 \mathrm{mg}$ first dose, then $75 \mathrm{mg}$ daily), Ticagrelor (180 mg first dose, then $90 \mathrm{mg}$ twice daily), atorvastatin ( $80 \mathrm{mg}$ first dose, then $80 \mathrm{mg}$ daily). Patients with atrial fibrillation were excluded from the study. Long lesions requiring overlapping stents were also excluded from our study. Direct stenting was tried in all cases. Predilatation at low pressure was done where there was difficulty in stent delivery. Infarct related artery only was addressed in our study and only single stent strategy group was included in this study. We have used thrombus aspiration catheter and Gp $11 \mathrm{~b} / 111$ a receptor blocker when there is huge thrombus load (TIMI thrombus grade 3 or more).Intracoronary adenosine and nicorandil were used as adjunctive pharmaco therapy to alleviate no flow. There were many patients where there was no flow even after mechanical thrombus aspiration and pharmacological vasodilator therapy. TIMI 111 flow was taken as successful recanalization criteria. Majority of our procedures $(70 \%)$ were done by radial route and rest (30\%) by femoral route.

Manual compression was done to secure haemostasis.

\section{Statistical analysis}

Instat 3 software was used for statistical analysis.

Fisher exact test was done to evaluate statistical significance between two groups.

\section{Results}

We have experienced 44 cases of no flow in our series. Incidence is around $7.75 \%$. Twenty six patients were suffering from diabetes. Incidence was $60 \%$. Thirty patients were male and fourteen were female patients. Male prevalence was $68 \%$ and female prevalence was $32 \%$ (Table 1). Twenty four patients were hypertensive. Incidence is about $54 \%$. Thirty two patients were smokers taking more than 10 cigarettes per day. Prevalence is around $72 \%$ and it was most common risk factor. Dyslipidaemia was seen only in sixteen patients and incidence was about $36 \%$.No flow was common in elderly patients.

Table 1: Baseline characteristics of no flow patients.

\begin{tabular}{|c|c|c|}
\hline Different category of no flow patients & No of Patients & Percentage (\%) \\
\hline Male & 30 & 68 \\
\hline Female & 14 & 32 \\
\hline Diabetes & 26 & 60 \\
\hline Dyslipidaemia & 16 & 36 \\
\hline Hypertension & 24 & 54 \\
\hline Smoker & 32 & 72 \\
\hline
\end{tabular}

Only four patients were below 40 years age group. Twelve patients were in the 40-60 years age group (Chart 1). Twenty patients were in the age group of $60-80$. Fourteen patients were in cardiogenic shock requiring pharmacological pressure support mainly noradrenaline, dobutamine and dopamine. We did not use mechanical circulatory support device in any of our patients. Amongst patients with cardiogenic shock there were ten patients having anterior wall STEMI and four patients having inferior wall STEMI. Amongst cardiogenic shock cases ten patients were diabetic. Incidence is $70 \%$. Majority of them were having multi vessel disease. Two patients died during hospital stay. They were suffering from anterior wall STEMI. We could not reestablish flow in those patients. They were having persistent cardiogenic shock.

Involvement of Left anterior descending artery (LAD) was in eighteen patients.

Right coronary artery (RCA) was culprit in twenty four cases. No flow was most common in RCA territory. Least common was left circumflex (LCX) artery (Chart 2). Only two cases were seen in LCX territory. Average diameter of RCA was greater than LAD. Most. RCA were tortuous, dilated and ecstatic.

Incidence of no flow was higher in long stents. Highest prevalence $(60 \%)$ was seen in stents with more $30 \mathrm{~mm}$ length.

Amongst failure cases two patients died in their hospital stay and they were having persistent cardiogenic shock.

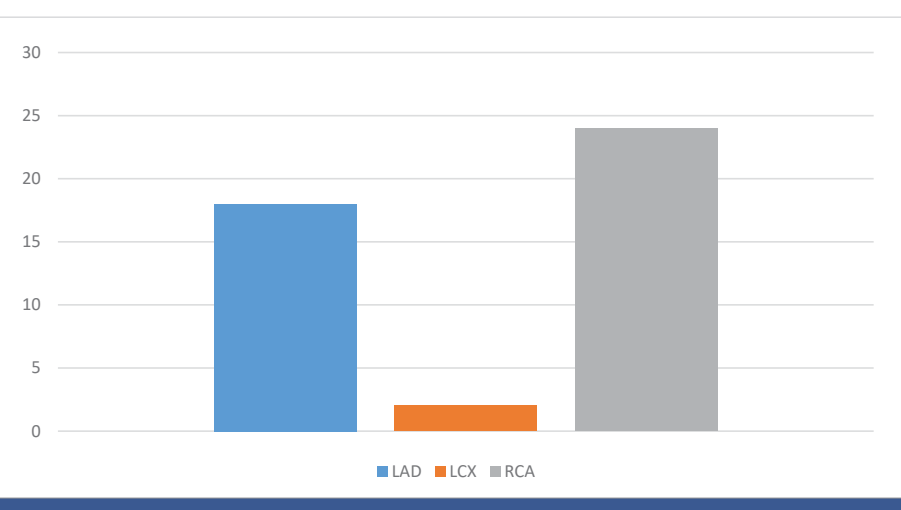

Chart 1: No flow in different coronary artery.

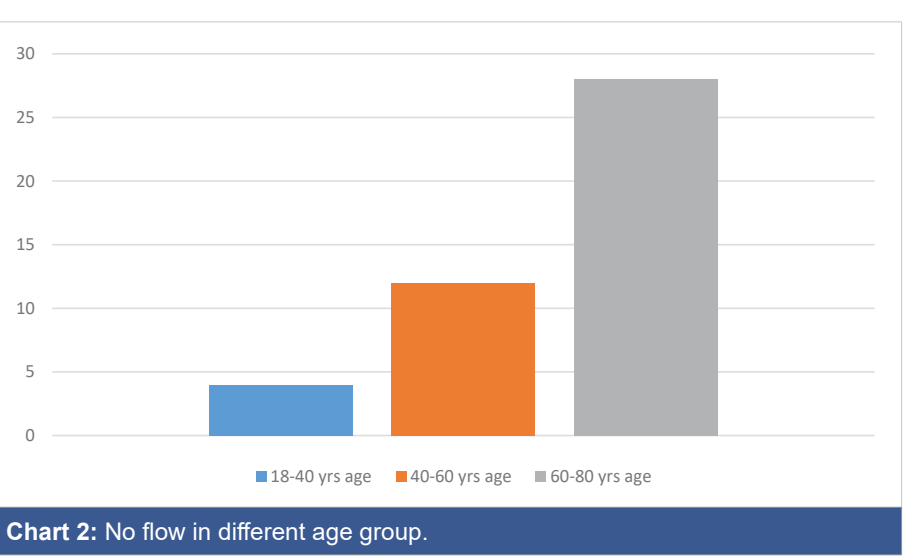


Another six patients died during thirty days. Two died in their home probably because of arrhythmia. Three died due to persistent heart failure later on. Another was admitted with heart failure but died due to incessant ventricular tachycardia refractory to recurrent electrical cardio version.

Thirty five patients died within one month in successful recanalization group.

Four morepatients died within one year again due to heart failure in no flow group. Seventy six patients died in successful recanalization group. One month mortality rate in no flow group was $50 \%$ and $6.25 \%$ in successful recanalization group (Table 2) (Chart3). One year mortality was $12.5 \%$ in successful recanalization group and 66\% in no flow group (Tables 3,4).

Table 2: Baseline characteristics of normal flow patients. Baseline category of normal flow patients $\quad$ No of patients Percentage (\%)

\begin{tabular}{|c|c|c|}
\hline Male & 426 & 80 \\
\hline Female & 110 & 20 \\
\hline Diabetes & 268 & 50 \\
\hline Dyslipidaemia & 188 & 35 \\
\hline Hypertension & 294 & 55 \\
\hline Smoker & 321 & 60 \\
\hline
\end{tabular}

Table 3: One month mortality rate comparison between successful recanalization and no flow group:

\begin{tabular}{|c|c|c|}
\hline & Death & Survived \\
\hline No Reflow group(44) & 8 & 36 \\
\hline Successful recanalization group(536) & 35 & 501 \\
\hline
\end{tabular}

Table 4: One year mortality rate comparison between successful recanalization and no flow group:

\begin{tabular}{|c|c|c|}
\hline & Death & Survived \\
\hline No Reflow group(44) & 12 & 32 \\
\hline Successful recanalization group(536) & 76 & 460 \\
\hline
\end{tabular}

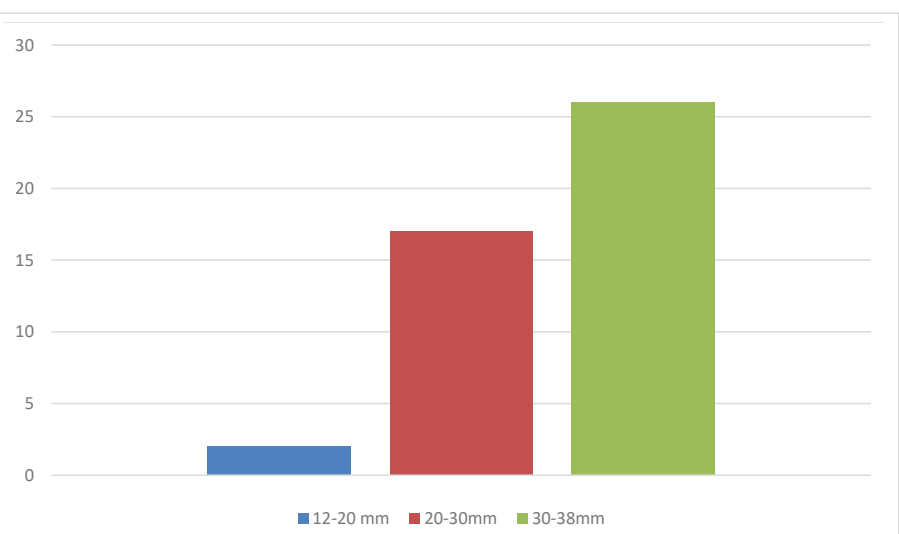

Chart 3: No flow and different stent length.

One month mortality rate in no flow group was 50\% and $6.25 \%$ in successful recanalization group. Relative risk of dying within one month in no-reflow group as compared to successful recanalization group is highly significant (RR is 8.00 ; $95 \%$ confidence interval 1.864 to 34.333 ; with a $p$ - value of 0.0027). One year mortality rate comparison between successful recanalization and no-reflow group:
Relative risk of dying within one year in no-reflow group as compared to successful recanalization group is statistically significant (RR is 5.333; 95\% confidence interval 1.961 to 14.504; with a $p$-value of 0.0009 ) (Chart 4).

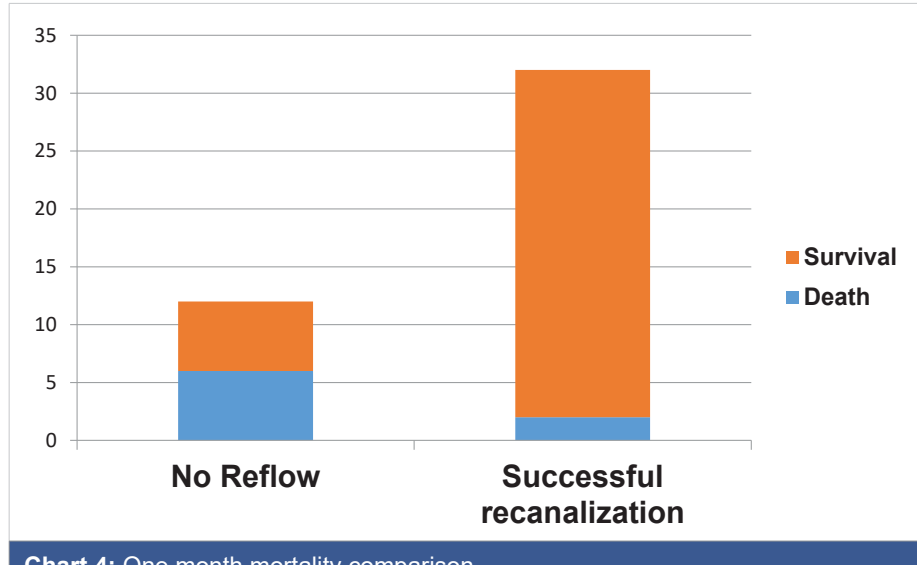

Chart 4: One month mortality comparison.

\section{Discussion}

Incidence of no flow is approximately $8.8-10 \%$ of patients undergoing primary percutaneous coronary intervention (PCI) in ST segment elevation myocardial infarction (STEMI). No flow is associated with increased incidence of major adverse cardiac events. No flow will cause adverse left ventricular remodeling due to poor wound healing, infarct extension and expansion. This will result in left ventricular systolic and diastolic dysfunction, increased propensity for different cardiac arrhythmias, heart failure and mortality.

No flow is accompanied by chest pain, electrical instability and possible hemodynamic compromise. The mechanisms of no flow are thrombus-plaque embolization, platelet activation, release of vasoconstrictors and vasospasm.

Thirty day mortality of refractory no flow is 32\% [3]. No flow is directly proportional to thrombus burden. Lot of STEMI patients have huge thrombus burden. After stent deployment or balloon dilatation thrombus may fragment and migrate distally in coronary artery. Routine use of aspiration catheter and Gp 11b/111 a receptor blocker in primary PCI is not recommended now. TASTE trial has proven that routine use of aspiration catheter is not beneficial and it increases incidence of stroke.

Incidence of no flow was $32 \%$ in a study by Rezkalla, et al. There were 347 STEMI patients in their series who had undergone primary PCI. Patients with no flow were treated pharmacologicallyby intracoronary nitroprusside,nicardipine, or verapamil. There was improvement in epicardial coronary blood flow as well as reduced incidence of no flow. Delayed presentation, hyperglycaemia and hypercholesterolemia were associated with increased incidence of no flow in their study. No flow is also more frequently seen in patients with female sex, hypertension, mild-to-moderate renal insufficiency and 
elevated inflammatory markers [5]. Intravascular ultrasound (IVUS) study revealed specific features like more soft yellow plaque and thrombus in lesion are associated with increased prevalence of no flow phenomenon [6]. Control of blood sugar and intensive statin therapy before procedure have been found beneficial to reduce no flow incidence $[7,8]$. Procedural factors like direct stenting, avoidance of high pressure stent deployment and thrombus aspiration (if huge thrombus burden)are shown to be effective in decreasing no flow incidence [9].

Incidence of no flow was around $7.75 \%$ in our study. Right coronary artery was most commonly involved and least common was left circumflex artery. Thrombus burden and vessel ectasia were two important predisposing factors for no flow.

There was statistically significant effect on mortality in no flow patients. Relative risk of dying within one month in no flow group as compared to successful recanalization group is highly significant (RR is 8.00 ; $95 \%$ confidence interval 1.864 to 34.333 ; with a $p$ - value of 0.0027 ). Relative risk of dying within one year in no flow group as compared to successful recanalization group is statistically significant (RR is 5.333; $95 \%$ confidence interval 1.961 to 14.504 ; with a $p$-value of 0.0009).

Infarct related artery is usually filled with huge thrombus burden specially in patients with late presentation. Manipulation of stents, balloons, other devices in thrombus laden artery will lead to distal embolization. Embolized particle will clog microcirculation, reduce myocardial perfusion, increase oxidative stress and myocyte injury. Incidence of macroscopic embolization is around $16 \%$.

Suboptimal tissue perfusion as evidenced by reduced TIMI frame count was observed in 20 to $40 \%$ of patients in spite of TIMI 3 flow restoration in epicardial coronary artery [10]. Different vasodilators agents have been tried to improve TIMI 3 flow rate and to reduce no flow phenomenon. These are sodiumnitroprusside, norepinephrine, nicorandil, verapamil and adenosine [11].

\section{Conclusion}

Refractory no flow during STEMI intervention is associated with increased incidence of major adverse cardiovascular events (MACE). There is no established strategy to solve this phenomenon.

\section{References}

1. Prasad S, Meredith IT. Current approach to slow flow and no Reflow. Cardiac Interventions Today. 2008; 43-49.

2. Triantafyllou K, Metaxopoulos $\mathrm{P}$, Babalis D. Primary percutaneous coronary intervention of an unprotected left main using mini-crush drug-eluting stents facilitated by intracoronary reteplase. Catheter Cardiovasc Interv. 2011; 77: 515-521.

3. Rezkalla SH, Dharmashankar KC, Abdalrahman IB, Kloner RA. Noreflow phenomenon following percutaneous coronary intervention for acute myocardial infarction: incidence, outcome, and effect of pharmacologic therapy. J Interv Cardiol. 2010; 23: 429-436.

PubMed: https://pubmed.ncbi.nlm.nih.gov/20819117/

4. Wang HJ, Lo PH, Lin JJ, Lee H, Hung S. Treatment of slow/no-reflow phenomenon with intracoronary nitroprusside injection in primary coronary intervention for acute myocardial infarction. Catheter Cardiovasc Interv. 2004; 63: 171-176.

PubMed: https://pubmed.ncbi.nlm.nih.gov/15390241/

5. Amano $\mathrm{H}$, lkeda $\mathrm{T}$, Toda $\mathrm{M}$, Okubo R, Yabe $\mathrm{T}$, et al. Plaque composition and no-reflow phenomenon during percutaneous coronary intervention of low-echoic structures in grayscale intravascular ultrasound. Int Heart J. 2016; 57: 285-291.

PubMed: https://pubmed.ncbi.nlm.nih.gov/27170472/

6. Iwakura K, Ito H, Ikushima M, Kawano S, Okamura A, et al. Association between hyperglycemia and the no-reflow phenomenon in patients with acute myocardial infarction. J Am Coll Cardiol. 2003; 41: 1-7. PubMed: https://pubmed.ncbi.nlm.nih.gov/12570936/

7. Li X, Yang Y, Hao Y, Yang Y, Zhao J, et al. Effects of pre-procedural statin therapy on myocardial no-reflow following percutaneous coronary intervention: A Meta-analysis. Chin Med J. 2013; 126:1755-1760. PubMed: https://pubmed.ncbi.nlm.nih.gov/23652063/

8. Grygier M, Araszkiewicz A, Lesiak M, Grajek S. Role of adenosine as an adjunct therapy in the prevention and treatment of no-reflow phenomenon in acute myocardial infarction with ST segment elevation: review of the current data. Kardiologia Poloska. 2013; 71: 115-120.

9. Higashi H, Inaba S, Nishimura K, Hamagami T, Fujita Y, et al. Usefulness of Adjunctive Pulse Infusion Thrombolysis After FailedAspiration for Massive Intracoronary Thrombus. Canadian J Cardiol. 2011; 27: 869- 869.

10. Rezkalla SH, Stankowski RV, Hanna J, Kloner RA. Management of No-Reflow Phenomenon in the Catheterization Laboratory. JACC: Cardiovasc Interv. 2017; 10: 215-223.

PubMed: https://pubmed.ncbi.nlm.nih.gov/28183461/ 